\title{
Dark-Pulse Propagation in Optical Fibers
}

\author{
D. Krökel, N. J. Halas, ${ }^{(a)}$ G. Giuliani, and D. Grischkowsky \\ IBM Watson Research Center, Yorktown Heights, New York 10598
}

(Received 8 September 1987)

\begin{abstract}
We report measurements of the reshaping of $0.3-$ psec dark pulses due to their passage through $10 \mathrm{~m}$ of single-mode optical fiber. The measurements were performed as a function of intensity and the observed strong reshaping agrees qualitatively with the predictions of the nonlinear Schrödinger equation which suggest that we have observed the formation of dark-pulse solitons.
\end{abstract}

PACS numbers: $42.30 .-\mathrm{d}, 41.10 . \mathrm{Hv}, 42.10 . \mathrm{Qj}$

Propagation of short optical pulses through singlemode optical fibers appears to be the best physical example of a system described by the nonlinear Schrödinger equation (NLSE). ${ }^{1-10}$ In the traditional and simplest description of the problem, the single-mode propagation permits the problem to be reduced to the equivalent problem of plane waves. ${ }^{1-7,9,10}$ The solutions of this equation divide into two regimes depending on the relative sign of the fiber's group-velocity dispersion (GVD), a combination of the material GVD and the fiber waveguide dispersion. In the region of negative GVD, it is possible to have optical soliton propagation, where for the lowest-order soliton the effects of the nonlinear index of refraction balance the pulse-broadening effect of GVD and the pulse can propagate without distortion. Higherorder soliton solutions show periodic pulse-reshaping behavior. These features have been investigated and confirmed in a beautiful series of experiments. ${ }^{4}$ In the region of positive GVD the combined effects of self-phase modulation (SPM) and GVD lead to a frequency and temporal broadening of the propagating optical pulse. The combined action of these two effects can lead to the formation of a flat-topped optical pulse with a linear frequency sweep. This behavior is the key to the operation of the optical-fiber pulse compressor. ${ }^{6}$

Another solution of the NLSE for positive GVD, which has been much discussed but never observed, is the "dark-pulse soliton." 1,5,7,9 In this case, one has a cw or a long optical pulse with a rapid intensity dip, "dark pulse." Because the sign of the SPM is reversed for the dark pulse, it becomes possible to balance GVD to allow the dark pulse to propagate without distortion. This picture has been challenged by recent theoretical work which claims that the full three-dimensional NLSE has no dark-pulse soliton solution for positive GVD. ${ }^{8}$ Independent of this theoretical controversy, there have been no previous experimental investigations of this important problem.

In this Letter we describe a series of measurements of 0.3-psec dark-pulse propagation through a $10-\mathrm{m}$ singlemode optical fiber. The observations were made as a function of the dark-pulse intensity, and good qualitative agreement was obtained with numerical solutions of the plane-wave NLSE. At the highest intensity, our observations showed the formation of two 0.6 -psec dark pulses from single 0.3-psec input dark pulses. According to our numerical calculations, these two observed dark pulses may indicate the formation of dark-pulse solitons. When these calculations were performed for the experimental fiber length, as well as for longer propagation distances, the calculated dark pulses had converged to the analytical description of dark-pulse solitons.

The experiment shown in Fig. 1 was made possible by the newly developed "ultrafast light-controlled optical fiber modulator."11 The operation of the modulator is based on the optical Kerr effect, and its main component is $15 \mathrm{~mm}$ of single-mode optical fiber. Driving the modulator by subpicosecond, compressed and amplified $6000-\AA$ dye-laser pulses, we produced 0.3 -psec holes (dark pulses) on the much longer duration (100 psec) second-harmonic (5320 $\AA$ ) yttrium aluminum garnet pulses. The driving pulse is shown in Fig. 2(a), the resulting dark pulse is Fig. 2(b), and the calculated dark pulse ${ }^{11,12}$ in Fig. 2(c) for the experimental conditions. The output pulses from the modulator were measured by cross correlation with the 0.3 -psec probing pulses obtained by a partial reflection from the beam of driving pulses. The linearly polarized beam of dark pulses from the modulator was coupled into a $10-\mathrm{m}$ single-mode, polarization-preserving optical fiber (Newport Research F-SPA). The resulting linearly polarized output beam from the fiber was recollimated and directed into the same cross correlator, where the beam of $0.3-$ psec probing pulses (suitably delayed) was used for the measurement. The $10-\mathrm{m}$ optical-fiber length was limited by the delay line of the cross correlator; because of beam-

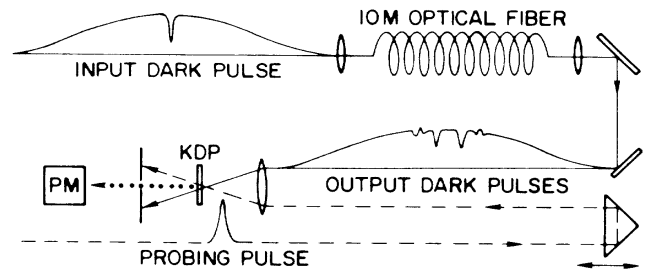

FIG. 1. Schematic diagram of the experiment. 


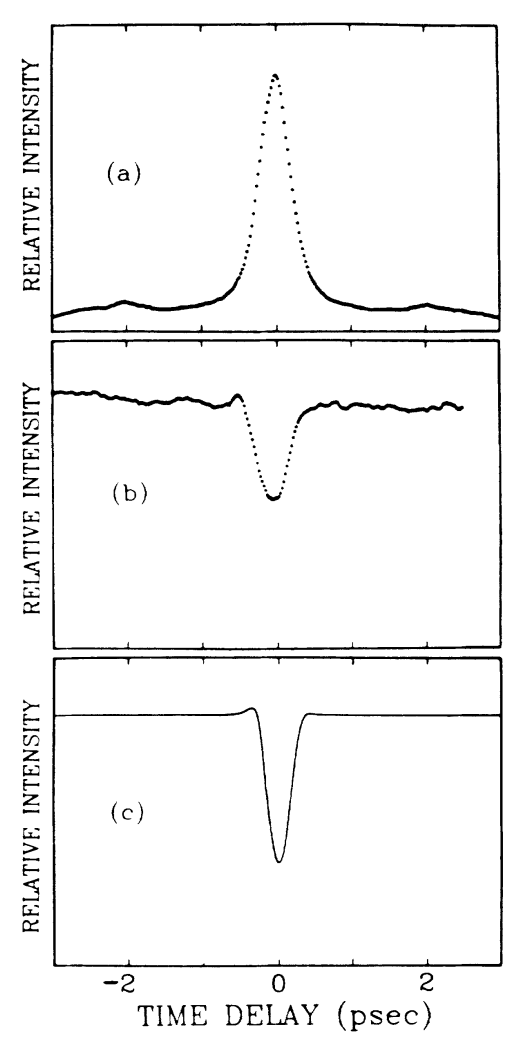

FIG. 2. (a) Autocorrelation measurement of the driving pulse. (b) Cross-correlation measurement of the input dark pulse. (c) Input dark pulse used in numerical integration of the nonlinear Schrödinger equation. For all traces, the base line is zero.

stability problems, further extensions significantly degraded the time resolution and reduced the signal-tonoise ratio. The cross-correlation signals were obtained by the noncollinear generation of sum-frequency light in a $300-\mu \mathrm{m}$-thick potassium dihydrogen phosphate crystal. The generated light was monitored by a photomultiplier connected directly to a boxcar integrator, which in turn was connected to a multichannel analyzer synchronized with the correlator delay. In order to reduce the effect of pulse-to-pulse fluctuations, the light pulse and the driving pulse were monitored at the output of the modulator by two photodiodes connected to discriminators driving a coincidence trigger. This arrangement triggered the boxcar amplifier only if the energies of both pulses were simultaneously within narrow preset energy windows. Usually twenty scans were averaged with a typical scan requiring about $100 \mathrm{sec}$.

The measured dark pulses from the $10-\mathrm{m}$ fiber are shown in Fig. 3 as a function of the coupled input power ${ }^{13}$ for the dark pulse of Fig. 2(b). As shown in Fig. 3(a), at low powers (under $0.5 \mathrm{~W}$ ) the output dark pulses have broadened by an order of magnitude, and a strong symmetric ringing structure has developed. At these power levels, nonlinear effects are negligible and

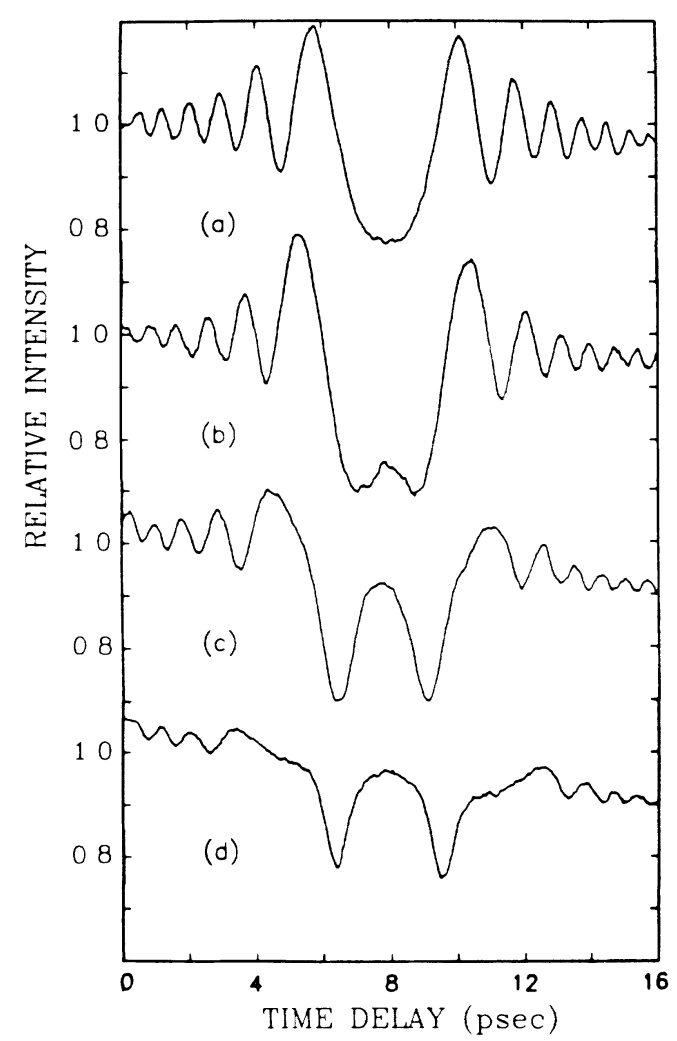

FIG. 3. Measured output dark pulses from the 10-m fiber vs peak coupled input power: (a) $0.2 \mathrm{~W}$, low intensity; (b) $2 \mathrm{~W}$; (c) $9 \mathrm{~W}$; (d) $20 \mathrm{~W}$.

the propagation can be described by linear dispersion theory. Therefore, a transform-limited dark pulse can be considered as the superposition of a constant cw background at the carrier frequency and an optical pulse out of phase by $\pi$ radians with respect to the cw field. In the fiber these two fields propagate independently. As the pulse propagates it broadens monotonically because of GVD. When the broadened pulse width becomes much greater than the initial pulse width, the reshaped pulse has acquired a linear frequency sweep as a result of the action of GVD. The observed intensity modulation is simply the interference between the broadened frequency-swept pulse and the carrier wave. The beat frequency increases quadratically from the center of the pulse to the wings, showing the quadratic time dependence of the phase corresponding to a linear frequency sweep. ${ }^{14} \mathrm{We}$ calibrated the broadening of this out-of-phase hypothetical pulse by propagating through the fiber at low intensity the light pulse simultaneously produced by the modulator. For a 0.3-psec input light pulse, we observed a 9psec output pulse. Given GVD $=0.044 \mathrm{psec} / \AA \cdot \mathrm{m}$ of the fiber, this gives a bandwidth of approximately $20 \AA$, indicating strong phase modulation compared with the transform limit of $11 \AA$.

When the coupled input power was increased to $2 \mathrm{~W}$, the output pulse developed a slight peak at the center in- 


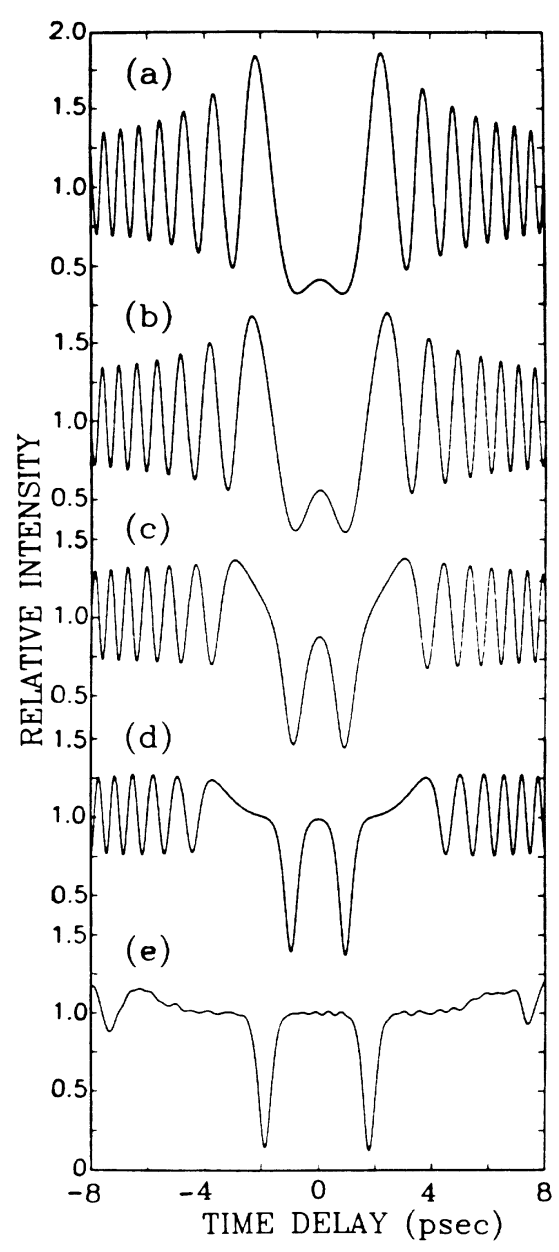

FIG. 4. Calculated output dark pulses from the fiber vs peak coupled input power and fiber length: (a) $0.2 \mathrm{~W}, 10 \mathrm{~m}$; (b) 4 $\mathrm{W}, 10 \mathrm{~m}$; (c) $18 \mathrm{~W}, 10 \mathrm{~m}$; (d) $40 \mathrm{~W}, 10 \mathrm{~m}$; and (e) $40 \mathrm{~W}$, $20 \mathrm{~m}$.

dicating a small phase shift at the carrier frequency caused by the nonlinearity. The output modulation pattern shifted slightly outwards from the center and was no longer described by a simple linear frequency sweep. Increasing the power to $9 \mathrm{~W}$ caused the central pulse to split into two clearly resolved dips, and the modulation on the wings was again pushed outwards. At $20-\mathrm{W}$ input power, the modulation was pushed completely out of the central region and two well-defined 0.6-psec dark pulses developed.

The above observations were studied by the numerical integration of the plane-wave NLSE (with use of a split step routine described in Refs. 1 and 12) through the $10-\mathrm{m}$ optical fiber starting with the input pulse shown in Fig. 2(c). In the low-intensity limit, we calculated the fiber output dark pulse of Fig. 4(a). Here, a welldeveloped symmetric ringing is obtained over a large time interval and is almost perfectly described by the interference between a broad weak pulse with a linear fre- quency sweep and the cw carrier wave. The experimental time resolution of 0.3 psec strongly attenuates the predicted higher-frequency modulation on the wings. In order to obtain better agreement with our observations in the nonlinear regime, the power (or equivalently the nonlinear index $n_{2}$ ) was increased by 2 times in the calculation compared with the experimental value. Given the difficulties in measuring peak instantaneous power, ${ }^{13}$ the uncertainty in $n_{2}=1.1 \times 10^{-13}$, and the problem of knowing the effective fiber core area because of the transverse intensity variation, ${ }^{5}$ we consider this adjustment to be within our experimental limits. With the input power raised to $4 \mathrm{~W}$, the calculated output pulse in Fig. 4(b) shows a slight increase of the center bump, caused by the nonlinearity. For $18-\mathrm{W}$ input pulses, the double-dip structure in the center of the pulse continues to develop into two separate pulses, while the amplitude of the ringing pattern is reduced together with a phase shift. Finally, with an input power of $40 \mathrm{~W}$, we calculate in agreement with experiment the double-pulse structure shown in Fig. 4(d). Because the soliton velocity depends on the modulation depth of the input pulse, the fact that the time separation between the pulses is shorter than observed is indicative of our experimental uncertainty in measuring the input dark-pulse modulation depth.

The following considerations led to our preliminary conclusion that the dark pulses in Fig. 4(d) and consequently Fig. 3(d) may represent the formation of darkpulse solitons. Initially, when the dark pulse enters the fiber, self-phase modulation causes a phase change that follows the pulse shape. However, the time-dependent phase $\phi(\xi)$ for the dark-pulse soliton has one of the two possible time dependences

$$
\sin \phi(\xi)= \pm A \tanh (\xi) /\left[1-A^{2} \operatorname{sech}^{2}(\xi)\right]^{1 / 2}
$$

over the $A^{2} \operatorname{sech}^{2}(\xi)$ dark pulse. ${ }^{1}$ Consequently, to first order the phase dependence and the input pulse shape are an approximate superposition of two $(+/-)$ solitons. Recent calculations have shown that a single dark pulse can evolve into two $(+/-)$ dark-pulse solitons which continue to move apart with propagation distance. ${ }^{9}$ The dark-pulse shapes in Fig. $4(d)$ are quite accurately fitted by a $0.6-\mathrm{psec} \operatorname{sech}^{2}(\xi)$ pulse shape. In addition, the calculated time-dependent phase also quite accurately fits the expression given above for the first $(-)$ soliton and the second delayed $(+)$ soliton. As a further indication that soliton formation has possibly occurred, we extended the numerical calculation to $20 \mathrm{~m}$ as shown in Fig. 4(e). Here the dark pulses further separated into two well-defined pulses, and the ringing on the wings was reduced in amplitude and pushed out in time. As expected for solitons, the pulse shapes did not change nor did the time-dependent phase. This convergence to the soliton solutions indicates that our experimental results are consistent with the formation of darkpulse solitons as predicted by the plane-wave nonlinear 
Schrödinger equation.

This research was partially supported by the U.S. Office of Naval Research.

${ }^{(a)}$ Present address: AT\&T Bell Laboratories, Holmdel, NJ 07733.

${ }^{1}$ A. Hasegawa and F. Tappert, Appl. Phys. Lett. 23, 142, 171 (1973).

${ }^{2}$ G. B. Whitham, Linear and Nonlinear Waves (Wiley, New York, 1974).

${ }^{3}$ Solitons in Action, edited by K. Lonngren and A. Scott (Academic, New York, 1978).

${ }^{4}$ L. F. Mollenauer, R. H. Stolen, and J. P. Gordon, Phys. Rev. Lett. 45, 1095 (1980).

${ }^{5}$ B. Bendow, P. Gianino, N. Tzoar, and M. Jain, J. Opt. Soc. Am. 70, 539 (1980).

${ }^{6}$ H. Nakatsuka, D. Grischkowsky, and A. C. Balant, Phys. Rev. Lett. 47, 910 (1981); D. Grischkowsky and A. C. Balant, Appl. Phys. Lett. 41, 1 (1982); A. C. Balant and
D. Grischkowsky, U.S. Patent No. 4588957 (1986).

${ }^{7}$ B. Crosignani, A. Cutolo, and P. DiPorto, J. Opt. Soc. Am. 72, 1136 (1982).

${ }^{8}$ D. N. Christodoulides and R. I. Joseph, Opt. Lett. 9, 229, 408 (1984).

${ }^{9}$ K. J. Blow and J. J. Doran, Phys. Lett. 107A, 55 (1985).

${ }^{10}$ H. G. Winful, in Optical Fiber Transmission, edited by E. E. Basch (Sams, Indianapolis, Indiana, 1986).

${ }^{11}$ N. J. Halas, D. Krökel, and D. Grischkowsky, Appl. Phys. Lett. 50, 886 (1987).

${ }^{12}$ N. J. Halas, Ph.D. thesis, Bryn Mawr College, 1986 (unpublished).

${ }^{13}$ This is the background power level corresponding to unity on the intensity scales of Figs. 3 and 4 . This power was obtained by the measurement of the energy of the 100-psec, $5320-\AA$ pulse with a photodiode, calibrated by measurement of the average power of the second-harmonic yttrium aluminum garnet pulse train with a calorimeter.

${ }^{14}$ J. E. Rothenberg and D. Grischkowsky, Opt. Lett. 12, 99 (1987). 